\title{
Development of newly designed VHF interferometer system for observing earthquake-related atmospheric anomalies
}

\author{
By Isao Yamamoto, ${ }^{* 1}$ Hironobu Fujiwara, ${ }^{* 2}$ Masashi Kamogawa, ${ }^{* 3, \dagger}$ Atsushi Irono, ${ }^{* 4}$ \\ Valeri Kroumov ${ }^{* 5}$ and Takashi AzAKAmi ${ }^{* 1}$ \\ (Communicated by Seiya UYEDA, M.J.A.)
}

\begin{abstract}
Temporal correlation between atmospheric anomalies and earthquakes has recently been verified statistically through measuring VHF FM radio waves transmitted beyond the line-of-sight. In order to locate the sources of such atmospheric anomalies, we developed a VHF interferometer system (bistatic-radar type) capable of finding the arrival direction of FM radio waves scattered possibly by earthquake-related atmospheric anomalies. In general, frequency modulation of FM radio waves produces ambiguity of arrival direction. However, our system, employing highsampling rates of the order of $\mathrm{kHz}$, can precisely measure the arrival direction of $\mathrm{FM}$ radio waves by stacking received signals.
\end{abstract}

Keywords: earthquake, atmospheric anomalies, interferometric observation, FM radio waves, bistatic radar

\section{Introduction}

Pre-seismic anomalous phenomena in the atmosphere and ionosphere as well as those in the telluric currents and ultra-low frequency electromagnetic (EM) waves have been reported since the 1970s. Gufeld et al. ${ }^{1)}$ explicitly pointed out the existence of pre-seismic sub-ionospheric anomalies by transmission observation of VLF electromagnetic waves propagating through the earth-ionosphere waveguide. This line of study has further been developed mainly in Russia, Japan, and Italy. Kushida and Kushida ${ }^{2}$ reported anomalous transmissions of VHF FM radio waves before earthquakes. They measured the FM radio waves from broadcast stations located beyond the line-ofsight. Also from the observation of VHF transmissions, pre-seismic atmospheric anomalies were statis-

*1 Department of Information and Computer Engineering, Okayama University of Science, Okayama, Japan.

*2 JSG High School, Tokyo, Japan.

*3 Department of Physics, Tokyo Gakugei University, Tokyo, Japan.

*4 Department of Fundamental Science, Okayama University of Science, Okayama, Japan.

*5 Department of Electronic Engineering, Okayama University of Science, Okayama, Japan.

$\dagger$ Correspondence should be addressed: M. Kamogawa, 4-1-1 Nukuikitamachi, Koganei, Tokyo 184-8501, Japan (e-mail: kamogawa@u-gakugei.ac.jp). tically verified by Fujiwara et al. . $^{3}$ In their paper, it was shown that the appearance of atmospheric anomalies lasting for a few minutes to several hours was significantly enhanced within 5 days before $\mathrm{M} \geq 4.8$ earthquakes. Spatial identification of such atmospheric anomalies is considered important in clarifying their causal relationship with impending earthquakes. For this purpose, an electromagnetic wave interferometer has been constructed to observe the arrival direction of electromagnetic waves scattered or reflected at the atmospheric anomalous region. When we observe FM radio waves by a VHF interferometer, frequency modulation of radio waves produces ambiguity of arrival direction as discussed later. Furthermore, according to Fujiwara et al., ${ }^{3}$ the intensity of relevant signals is very low, i.e., only several decibel $(\mathrm{dB})$ enhancement from the background ranging from -105 to $-95 \mathrm{dBm}$. In the present work, we have tried to construct a VHF interferometer system which can detect arrival direction of FM radio waves at very low level of intensity.

\section{System configuration}

Radio interferometry to measure the arrival direction of electromagnetic waves is a well established technology (See text book written by Schilling. $\left.{ }^{4}\right)$ ). An interferometer, working like a bistatic radar, provides the direction to the reflecting and scattering 


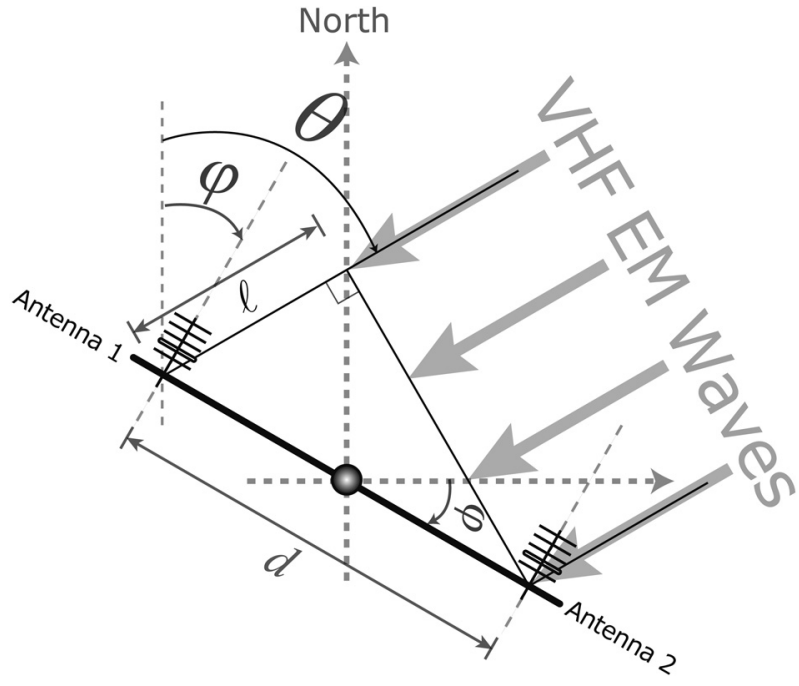

Fig. 1. A relation between the arrival angle and the direction of the rotating antennas. North is directed upward in this figure.

point of the atmosphere and ionosphere. In the VHF band, for example, it is frequently used to locate the meteors plunging into the ionosphere. ${ }^{5)}$ In the present study, the similar methodology is used to find the location of atmospheric anomalies possibly associated with earthquakes.

The relation between the arrival angle of the VHF EM waves and the direction of the rotating antennas is shown in Fig. 1. The arrival angle $\theta$ from north is given by

$$
\theta=\sin ^{-1}\left(\frac{\lambda}{d} \cdot \frac{\delta}{2 \pi}\right)+\varphi
$$

where $\delta$ is phase difference of electromagnetic waves received at two antennas, $\varphi$ is the angle of rotating antennas from the north, $\lambda$ is the wave length of the VHF waves, and $d$ is the distance between the two horizontal antennas.

The block diagram of our interferometer system for azimuth detection is shown in Fig. 2. The system consists of the following parts: 1) A pair of rotating Yagi-Uda antennas, 2) $30 \mathrm{~dB}$ pre-amplifiers, 3) receivers (e.g. commercial FM digital tuners) connected to a local oscillator used in common with $60 \mathrm{~dB}$ operational amplifier, 4) $20 \mathrm{~dB}$ amplifier, 5) two phase difference analyzers using dedicated ICs (Analog Devices; AD8302), and 6) a signal meter.

Characteristic curves of output voltages of the phase difference analyzers are shown in Fig 3. Figure 3a shows phase-difference angle $\delta$ of the input EM waves and the output voltages $\left(V_{\mathrm{Ph} 1}\right.$ and $\left.V_{\mathrm{Ph} 2}\right)$. Since the output voltage from one analyzer yields two candidates of phase difference angles as shown by the solid line in Fig. 3a, another analyzer operating with a $\pi / 2$ phase shifter is required to determine the unique phase angle. The characteristic curve of the output voltage $V_{\mathrm{Ph} 2}$ is consequently 90 degrees shifted from that of $V_{\mathrm{Ph} 1}$. From the two output voltages, the characteristic curves showing the relation between the intensity of input EM wave and the output voltage at each phase angle are obtained as shown in Fig. 3b. As seen in this figure, the system is finally so designed that $-116 \mathrm{dBm}$ can be detectable when the pre-amplifier and the amplifier are integrated in the system. In order to obtain the relationship between the phase difference angle and two output voltages,

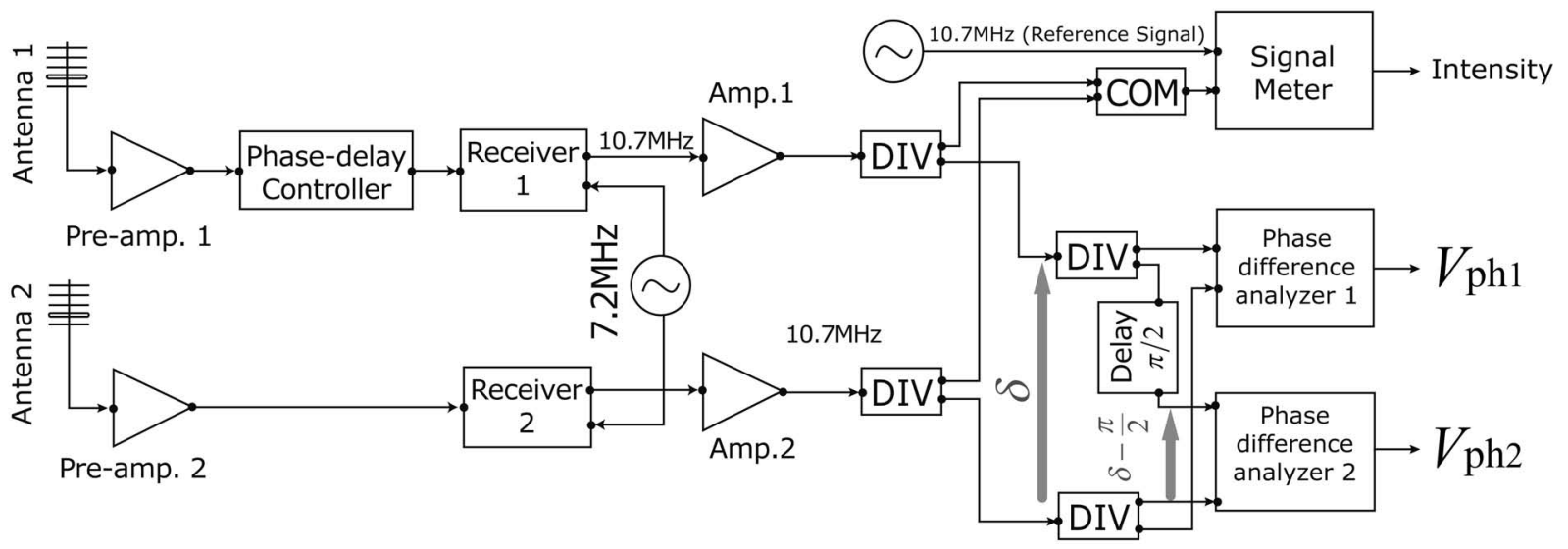

Fig. 2. Simplified block diagram of the VHF interferometer system. 


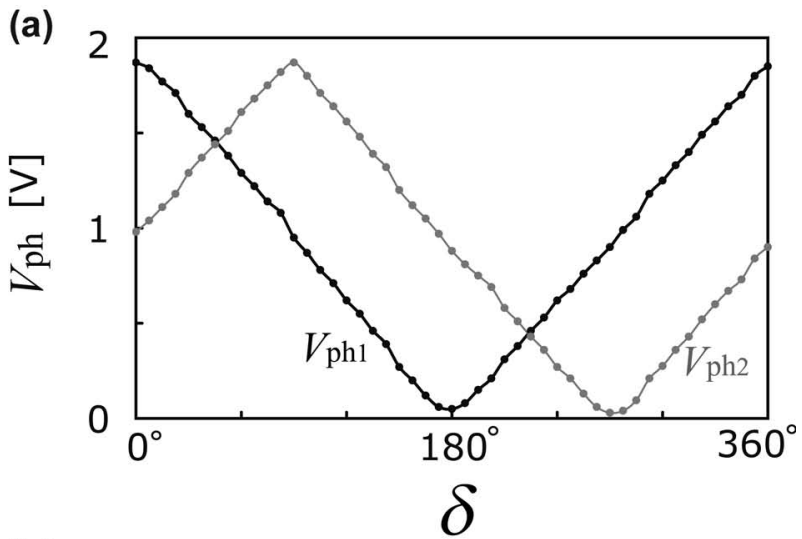

(b)

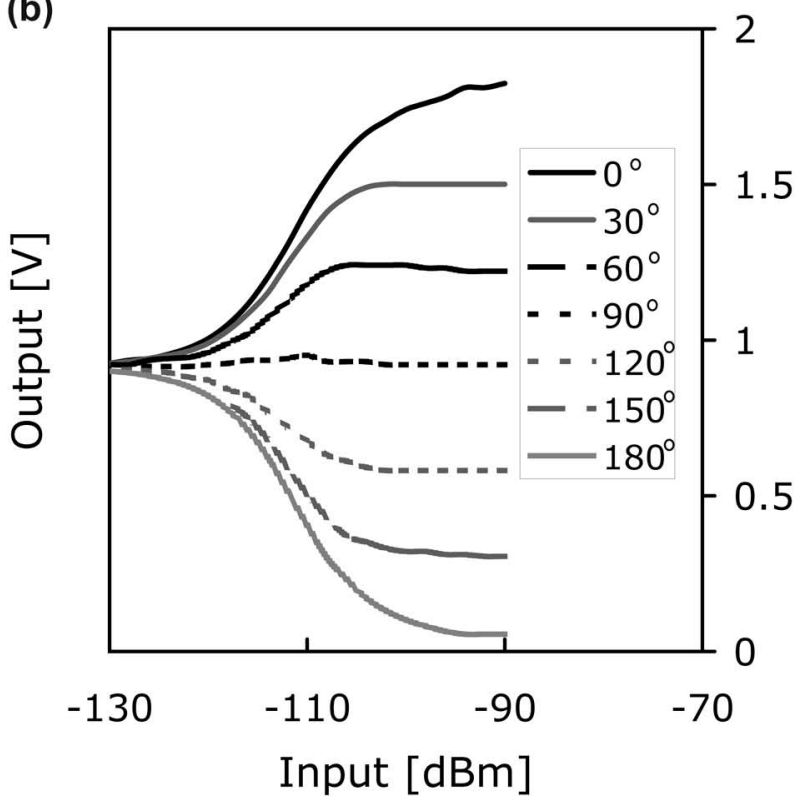

Fig. 3. (a) Characteristic curves of phase-difference angle of input VHF waves versus output voltages. (b) Dependence of output voltage on input intensity at seven phase differences $(\delta=0,30,60,90,120,150$, and 180 degrees $)$.

a Lissajous figure of phase chart indicating $V_{\mathrm{PH} 1}$ and $V_{\mathrm{PH} 2}$ is introduced as shown in Fig. 4.

Figure 5 shows the temperature dependence of FM radio receivers. Although commercial FM digital tuners are normally very cheap and measure the intensity accurately, they produce phase difference due to serious temperature-dependent property of super-heterodyne circuit. Therefore, if a commercial FM digital tuner is used, the temperature should be kept constant. However, if we use a receiver with a builtin, double balanced mixer (DBM) ${ }^{7)}$ this problem can be avoided as shown by solid dots in Fig. 5 .

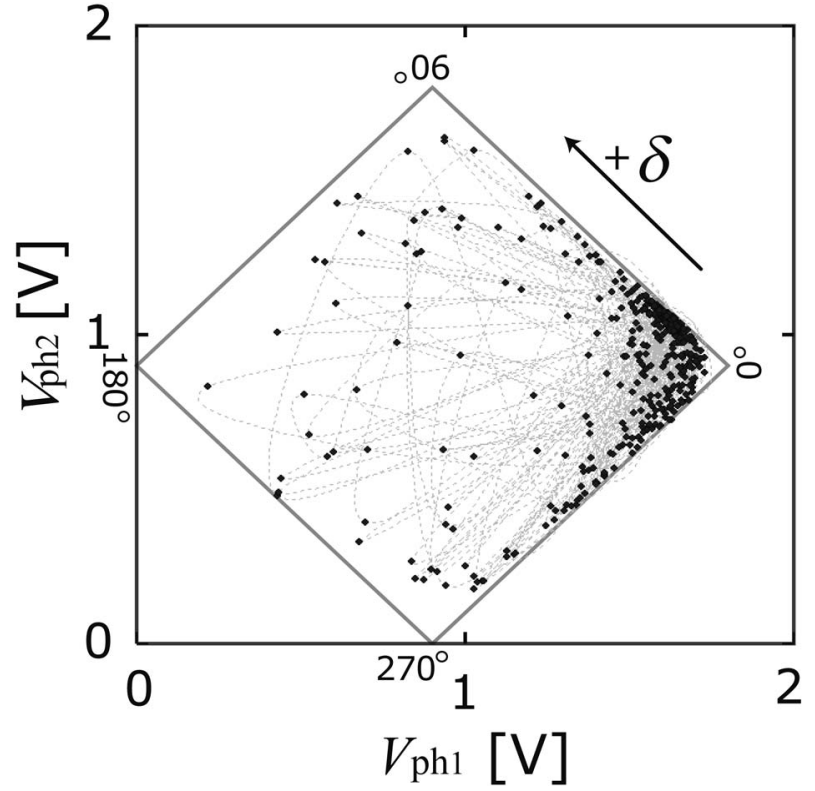

Fig. 4. Phase chart showing phase difference angles derived from two output voltages $V_{\mathrm{Ph} 1}$ and $V_{\mathrm{Ph} 2}$. Dots show data sampled in sequence as connected by thin dotted lines. This time-series is for the first one minute of the ten-minute data shown in Fig. 7.

\section{Calculation of arrival direction}

When we monitor FM radio waves, the frequency modulation of VHF EM waves degrade the accuracy of measurements, because the ceramic filters inside the intermediate frequency amplifier circuit normally have slight but significant individually different input-output characteristics which can cause large output phase difference $\delta$. The uncertainty of arrival angle $\theta$ consequently becomes very large. In order to find the way to obtain precise arrival direction, the following field experiment was conducted at 13:33:21 (LT) on April 18, 2007. A receiver was installed at Koganei, Tokyo (N35.7048, E139.4903). Five FM radio waves with different frequencies (76.0, $77.1,80.0,81.3$, and $82.5 \mathrm{MHz}$ ), emitted at $10 \mathrm{~kW}$ from the Tokyo Tower (N35.6586, E139.7454; $26 \mathrm{~km}$ distance) were measured as depicted in Fig. 6. The actual angle to the FM radio stations (i.e., Tokyo Tower) was $\theta_{\text {Trans. }}=120.8^{\circ}$. The interval between the two rotating antennas was set to $2.0 \mathrm{~m}$. The elevation angle was fixed at 10 degrees to obtain less interference (See Yamamoto et $\left.a l^{6}{ }^{6}\right)$. The rotating antennas were 5-element Yagi-Uda type (FM-5E, Maspro, Japan). In this experiment, commercial FM 


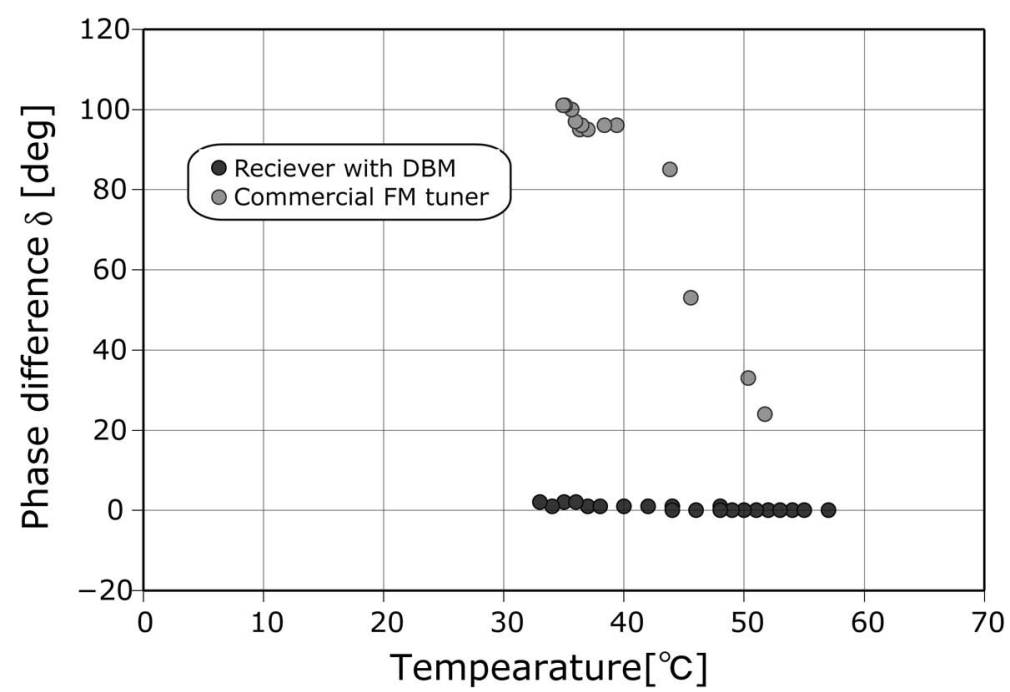

Fig. 5. Temperature dependence of output phase difference when a commercial FM radio tuner (T-422M, ONKYO, Japan) and a receiver with double balanced mixers (DBM) are used.

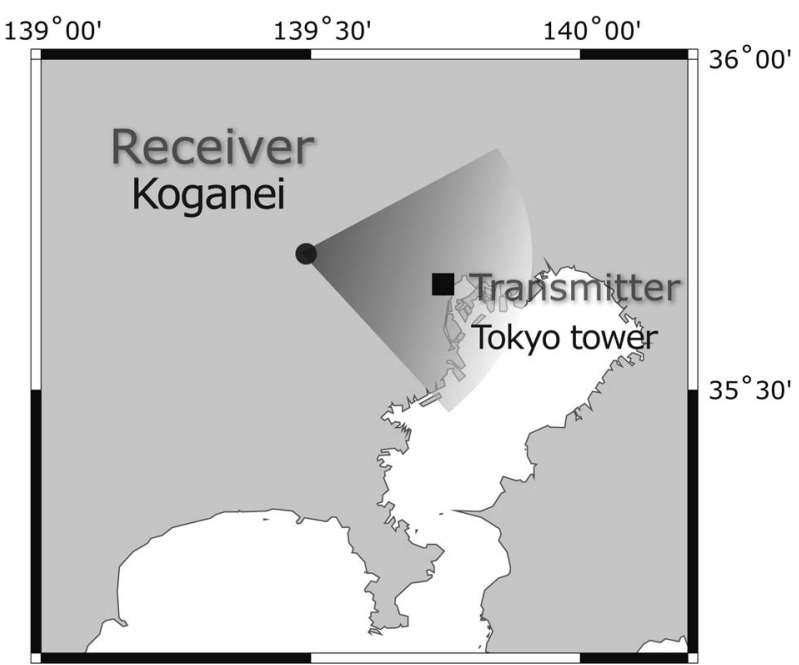

Fig. 6. Observation map. Usable antenna angles (Sector area) were obtained from the result shown in Fig. 9.

digital tuners (ST-GT550, Technics, Japan) were used. As shown in Fig. 7, the scatter of the estimated arrival direction of FM radio waves was very large. However, it was found that the median of 100 samplings converged to the exact transmitter direction (Fig. 7). Median values of different data number in four sampling frequencies are shown in Fig. 8. Figure 8 shows that if the median of 200 data is taken from $1000 \mathrm{~Hz}$ sampling, resulting $5 \mathrm{~Hz}$ data provide a few degrees of error. With the high-sampling rate of the order of $\mathrm{kHz}$, even the arriving direction of $\mathrm{FM}$ radio

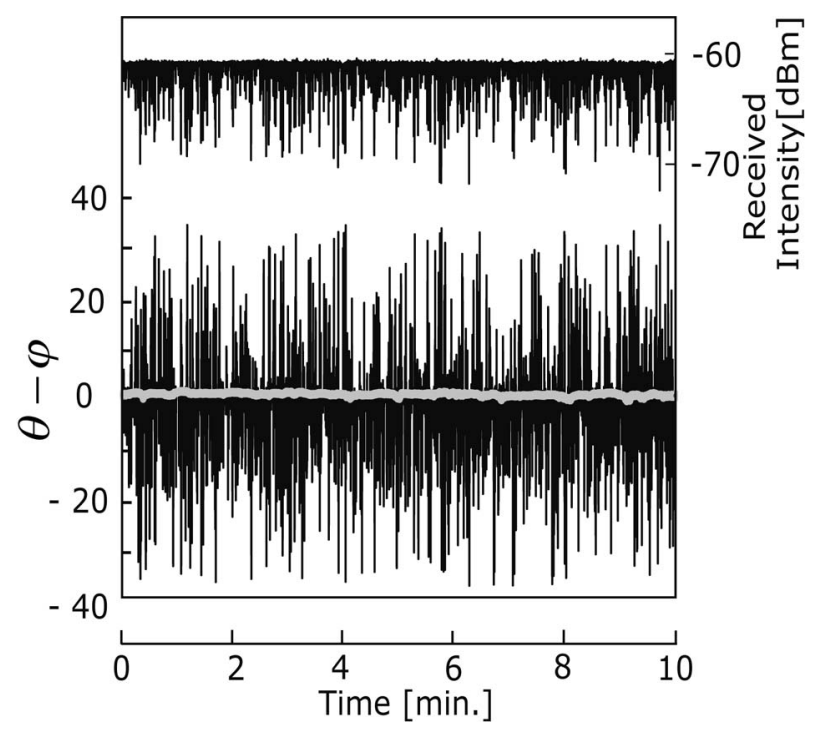

Fig. 7. Example time-series of received intensity (Upper panel) and direction (Lower). The observation and sampling frequencies were $77.1 \mathrm{MHz}$ and $10 \mathrm{~Hz}$. Solid lines and horizontal gray line in the lower panel are 10-minute raw data and running-median of 100 data.

waves from meteor-shower and lightning as well as from pre-seismic anomalies is expected to be determined.

When the angle of rotating antennas was changed from $\varphi=0$ to 360 degrees, tuned at the five target FM radio frequencies transmitted from the Tokyo Tower, observed arrival direction, $\theta-\varphi$, was as 


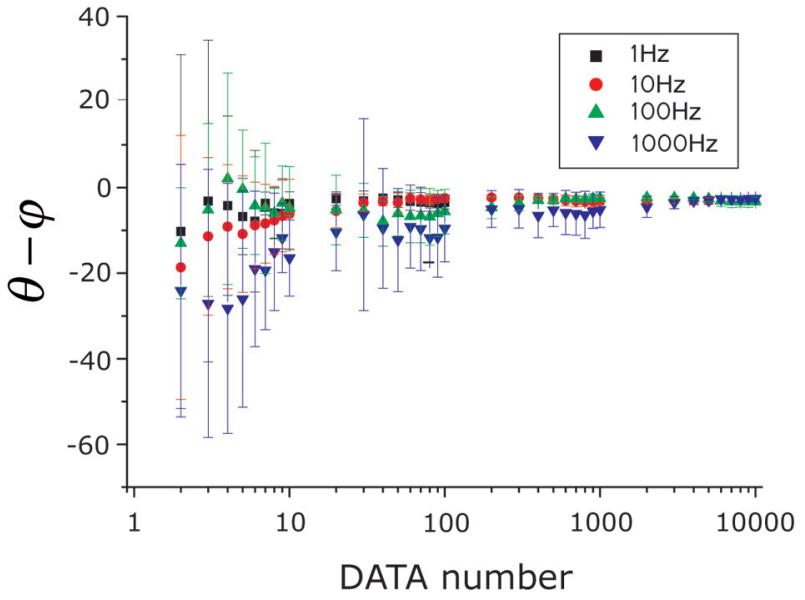

Fig. 8. Median values and error bars (derived from first and third quartile) against data number in four sampling frequencies $(1,10,100$, and $1000 \mathrm{~Hz})$.

shown in Fig. 9. From this result, it can be seen that the usable range of the antenna angle $\theta_{\text {Trans. }}-\varphi$ is from -40 to 40 degrees.

\section{Discussion}

It was confirmed that the direction of the source of the VHF FM radio wave can be obtained with sufficient accuracy by the present system. On the other hand, the enhancement of earthquake-related transmission anomalies was only at several $\mathrm{dBm}$ from the background level ranging from -105 to $-95 \mathrm{dBm}$, according to Fujiwara et al. ${ }^{3}$ From the results shown in Fig. 3b, however, the arrival direction of FM radio waves scattered or reflected by the earthquake-related atmospheric anomalies is expected to be measurable. The present system using dedicated IC operates stably for the high-sampling long-term monitoring. In general interferometer observations, non-directional antennas are used. The present study, however, uses the 5-element directional Yagi-Uda antennas to avoid the interference with the waves from other FM radio stations.

The present study only deals with the azimuth angle. However, the elevation angle is also measurable if one antenna, one receiver involving amplifiers, and one set of phase difference analyzers are added.

\section{Conclusion}

We developed a bistatic-radar type interferometer system to measure the arriving direction of FM

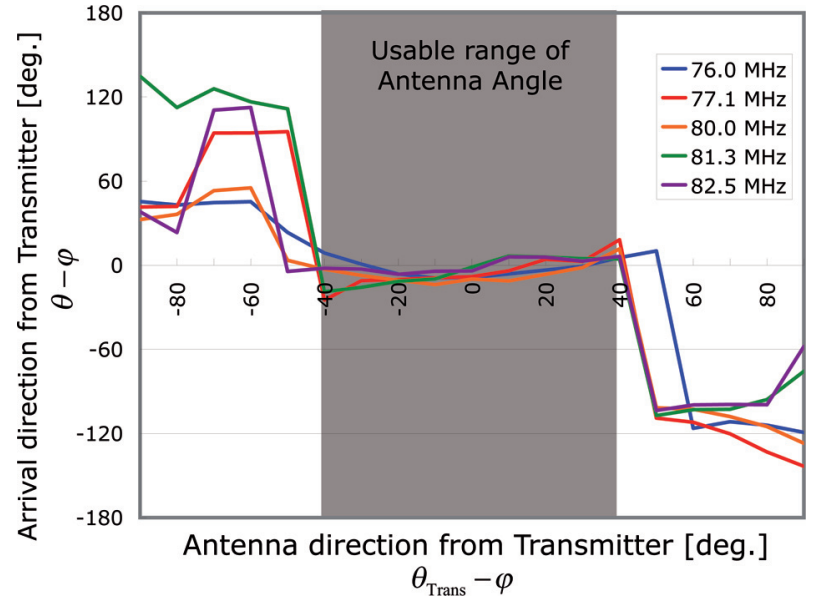

Fig. 9. The relations between the arrival angle $\theta$ of the FM wave and the direction angle $\varphi$ of the rotating antennas $(d=2.0 \mathrm{~m})$. Within -40 to 40 degree of antenna direction from transmitter, the arrival direction can be correctly assessed.

radio waves as weak as those possibly associated with impending earthquakes. Although frequency modulation of the FM radio waves produces ambiguity in the measurements of arrival directions, it has been demonstrated that the system we have developed, using very high sampling rates, can overcome this difficulty with resulting errors of a few degrees. This device, therefore, is expected to be able to identify also the direction to the atmospheric anomalies, which are supposed to cause the pre-seismic transmission of FM waves beyond the line-of-sight. Furthermore, with a couple of the present systems operating at distance, we will be able to triangulate the location of the pre-seismic anomalies.

\section{Acknowledgments}

Authors are also grateful to Professor Seiya Uyeda (M.J.A.) for useful comments. This research was partially supported by the Ministry of Education, Culture, Sports, Science and Technology, Grant-inAid for Young Scientists (B), No. 21710180, 2009 (M. K.), and Ito Science Foundation, 2007 (M. K.).

\section{References}

1) Gufeld, I. L., Rozhnoi, A. A., Tyumensev, S. N., Sherstuk, S. V. and Yampolsky, V. S. (1992) Radiowave disturbances in period to Rudber and Rachinsk earthquakes. Phys. Solid Earth 28, 267-270.

2) Kushida, Y. and Kushida, R. (2002) Possibility of earthquake forecast by radio observation in the VHF band. J. Atmos. Electr. 22, 239-255. 
3) Fujiwara, H., Kamogawa, M., Ikeda, M., Liu, J. Y., Sakata, H., Chen, Y. I. et al. (2004) Atmospheric anomalies observed during earthquake occurrences. Geophys. Res. Lett. 31, L17110, doi:10.1029/ 2004GL019865.

4) Schilling, D. L. (1986) Principles of Communications Systems (2nd ed.). McGraw Hill, New York.

5) Jones, J., Webster, A. R. and Hocking W. K. (1998) An improved interferometer design for use with meteor radars. Radio Sci. 33, 55-65.
6) Yamamoto, I., Kuga, K., Okabayashi, T. and Azakami, T. (2002) System for earthquake prediction research in the region of VHF frequency band. J. Atmos. Electr. 22, 267-275.

7) David, M. P. (2000) Microwave and RF Design of Wireless Systems. John Wiley \& Sons, Inc., New York, pp. 11-26 and pp. 225-246.

(Received Oct. 14, 2009; accepted Nov. 4, 2009) 